Pacific Journal of Mathematics

COMMUTATIVE ENDOMORPHISM RING 


\title{
COMMUTATIVE ENDOMORPHISM RINGS
}

\author{
S. H. Cox, JR.
}

Two problems of W. V. Vasconcelos are partially solved: (1) The total quotient ring of a commutative noetherian ring $R$ is quasi-frobenius if and only if $\operatorname{End}_{R}(A)$ is commutative for each ideal $A$ of $R$. (2) Let $R$ be a commutative quasiregular ring and $E$ a finitely presented $R$-module. If $E$ is faithful and $\operatorname{End}_{R}(E)$ is semi-prime, then $E$ is isomorphic to an ideal of $R$. Only commutative rings with unit and unital modules are considered.

1. In [3] Vasconcelos considers problems concerning commutative endomorphism rings. Toward the end he asks for a characterization of rings $R$ for which End $(A)$ is commutative for each ideal $A \subset R$. He conjectures the following answer for noetherian rings.

THEOREM 1.1. Let $R$ be a noetherian ring with total quotient ring T. If $\operatorname{End}_{R}(A)$ is commutative for each ideal $A$ of $R$ then $T$ is quasi-frobenius.

Proof. It is sufficient to show that for each maximal ideal $p$ of $T$ the local ring $S=T_{p}$ has Krull dimension zero and $\operatorname{Ann}_{S}(q)$ is a one dimensional $S / q$-vector space where $q=p S$ [2, Theorem 221]. Each ideal $I$ of $S$ has a commutative endomorphism ring since we can select $J \subset R$ such that $J \otimes S=I$ and observe that the natural map $\operatorname{End}_{R}(J) \otimes_{R} S \rightarrow \operatorname{End}_{S}\left(J \otimes_{R} S\right)$ is a ring isomorphism [1, p. 39, Proposition 11]. Also because $R$ is noetherian $q=\operatorname{Ann}_{S}(a)$ for some $a \in S$ [2, Theorem 82]. Since $\operatorname{End}_{S / q}\left(\operatorname{Ann}_{S}(q)\right)=\operatorname{End}_{S}\left(\operatorname{Ann}_{S}(q)\right)$ is commutative and $\operatorname{Ann}_{S}(q) \neq 0$, then $\operatorname{Ann}_{S}(q)$ is a one dimensional $S / q$ vector space. It remains to show $S$ is zero dimensional, i.e., $q$ is nilpotent. Since $a \neq 0$, then there is an integer $n$ such that $a \notin q^{n}$ [2, Theorem 79]. Since $\mathrm{Ann}_{S}(q)$ is simple we must have $S a=\operatorname{Ann}_{S}(q)$ and $q^{n} \cap S a=0$. Now we show $q^{n}=0$. Suppose not, choose $b \in q^{n}, b \neq 0$. Then $\operatorname{Ann}_{S}(b) \subset q=\operatorname{Ann}_{S}(a)$. Thus the correspondence $x b \rightarrow x a$ defines an $S$-homomorphism $f: S b \rightarrow S a$. Let $J=S a+S b$. This sum is direct since $S a \cap S b \subset S a \cap q^{n}=0$. Let $u, w \in \operatorname{End}_{S}(J)$ be the following composites:

$$
\begin{aligned}
& u: J \longrightarrow S a \subset J \\
& w: J \longrightarrow S b \stackrel{f}{\longrightarrow} S a \subset J .
\end{aligned}
$$

Then $u w(b)=a$ and $w u(b)=0$ contradicting the commutativity of $\operatorname{End}_{S}(J)$. Thus $q^{n}=0$, and $S$ is zero dimensional. 
The converse to 1.1 is true also. This is because $T$ is an injective $T$-module if $T$ is quasi-frobenius. Indeed if $R$ is any commutative ring whose total quotient ring $T$ is $T$-injective then for $A \subset R, \operatorname{End}_{R}(A)$ can be viewed as a subring of $\operatorname{End}_{T}(A T)$ which is a homomorphic image of $T$ and therefore commutative.

The next proposition gives a sufficient condition on $A \subset R$ for End $(A)$ to be commutative.

Proposition 1.2. Let $R$ be a commutative ring and $A$ an ideal of $R$. If $A \cap \operatorname{Ann}(A)=0$, then $\operatorname{End}_{R}(A)$ is commutative.

Proof. Let $f, g \in \operatorname{End}_{R}(A)$ and $c=f g-g f$. For $a, b \in A$ we have $a c(b)=c(a b)=f(g(a b))-g(f(a b))=f(a g(b))-g(b f(a))=g(b) f(a)-f(a) g(b)=0$. Hence $A c(A)=0$ implies $c(A) \subset A \cap \operatorname{Ann}(A)=0$. Therefore End $(A)$ is commutative.

An $R$-algebra will be called semi-prime if it has no non-zero nilpotent elements.

CoROLlary 1.3. If $R$ is semi-prime then End $(A)$ is commutative and semi-prime for each ideal $A \subset R$.

Proof. $A \cap$ Ann $(A)$ consists of nilpotents so End $(A)$ is commutative by 1.2. If $f \in \operatorname{End}(A)$ is nilpotent, say $f^{n}=0$, then for $x \in A \quad 0=f^{n}\left(x^{n}\right)=(f(x))^{n}$. Since $R$ is semi-prime $f(x)=0$ for $x \in A$. Thus $f=0$.

If $R$ is an integral domain, we can characterize the ideals of $R$ as those torsionless $R$-modules $E$ having End $(E)$ commutative. For if $x \in E \quad x \neq 0$ there is $f: E \rightarrow R$ with $f(x) \neq 0$. Let $y \in E$. The two homomorphisms $z \rightarrow f(z) x$ and $z \rightarrow f(z) y$ commute. Hence $f(y) x=f(x) y$, so $f(y)=0$ implies $y=0$. Thus $f$ is injective.

The next section is concerned with how well the property End $(A)$ is semi-prime distinguishes the ideals of a semi-prime ring $R$ from other $R$-modules.

2. In [3] Vasconcelos proves that when $R$ is noetherian and semi-prime a finitely generated faithful $R$-module $E$ with $\operatorname{End}_{R}(E)$ commutative and semi-prime is isomorphic to an ideal of $R$. He conjectures that the result may remain valid for a finitely presented $E$ even if $R$ is not noetherian. I could not resolve this but generalize his result to include those rings having an absolutely flat total quotient ring (called quasi-regular rings). The methods make no use of the commutativity of End $(E)$. Thus in the situation considered (in 2.2 below) semi-prime implies commutativity. Although we are con- 
sidering only commutative rings here, our generalization, unlike the original version of the theorem, can at least be conjectured for noncommutative rings.

This is the first step in the proof:

THEOREM 2.1. Let $R$ be a ring and $E$ a finitely present $R$-module. If $x \in E$ is nonzero, then there exists $f \in \operatorname{End}(E)$ nonzero such that $f(E) \subset R x$.

Proof. First suppose $R$ is noetherian. Let $p$ be a prime minimal over Ann $(x)$. Then there exists $y \in R x$ such that $p=\operatorname{Ann}(y)$ [2, Theorem 86]. Localize at $p$. Let $K=R_{p} / p_{p}$. Since $E_{p} \neq 0, E_{p} \otimes K$ is at least one dimensional by the Nakayama lemma [2, Theorem 78]. Thus there is a surjection $h: E_{p} \otimes K \rightarrow K$. As an $R_{p}$-module, $K=(R y)_{p}$. Let $g$ be the composite $E_{p} \rightarrow E_{p} \otimes K \stackrel{h}{\rightarrow}(R y)_{p} \subset(R x)_{p}$. Since $E$ is finitely presented, we have $\operatorname{Hom}_{R}(E, R x)_{p} \cong \operatorname{Hom}_{R_{p}}\left(E_{P}, R x_{P}\right)$ [1, p. 39, Proposition 11]. Hence $g=f / s$ for some $f: E \rightarrow R x$ and $s \in R \mid p$. Clearly, $f$ has the required properties. Thus the result holds when $R$ is noetherian. Since $E$ is finitely presented we can use the following well known technique to reduce to the noetherian case: Let $R^{m} \stackrel{A}{\rightarrow} R^{n} \stackrel{B}{\rightarrow}$ $E \rightarrow 0$ be a presentation of $E$, select bases $f_{i}, e_{j}$ : let $A\left(f_{i}\right)=\sum a_{i j} e_{j}$ $B\left(e_{j}\right)=m_{j}, x=\sum x_{j} m_{j}$ with $a_{i j}, x_{j} \in R$. Let $S$ be the subring generated by 1 and all the $x$ 's and $a$ 's. $S$ is noetherian by the Hilbert Basis Theorem. Let $F$ be the $S$-submodule generated by the $m$ 's. Then $F \otimes_{S} R=E$ and $x \in F$. Since $S$ is noetherian there is nonzero $g: F \rightarrow S x$. Tensoring with $S$ yields a commutative diagram:

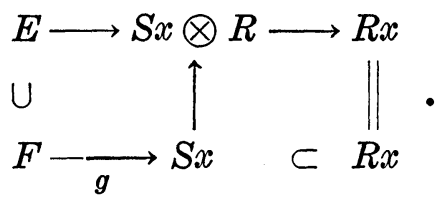

Hence we can take $f$ to be the composite of the maps on the upper row.

For an ideal $I$ of $R$ let Min $(I)$ denote the primes of $R$ minimal over $I$. For an $R$-module $E$ let Ass $(E)$ denote the Bourbaki associated primes of $E$. Thus Ass $(E)$ is the union over $x \in E$ of the sets $\operatorname{Min}(\operatorname{Ann}(x))$.

THEOREM 2.2. Let $R$ be a semi-prime ring, $E$ a finitely presented $R$-module. If End $(E)$ is semi-prime, then End $(E)$ is commutative and $\operatorname{Ass}(E)=\operatorname{Min}(\operatorname{Ann}(E))$.

Proof. For any finitely presented $R$-module $E \operatorname{Min}\left(\operatorname{Ann}_{R}(E)\right) \subset$ Ass $(E)$ and the mapping End $(E) \rightarrow \prod_{p \in A s s(E)} \operatorname{End}_{R_{p}}\left(E_{p}\right)$ induced by 
End $(E) \rightarrow \operatorname{End}_{R_{p}}\left(E_{p}\right)$ is an injective ring homomorphism. Thus it is sufficient to establish that if $p \in \operatorname{Min}(\operatorname{Ann}(x))$ and $x \in E$, then $E_{p}=$ $(R x)_{p}$. For then $\left[\operatorname{End}_{R}(E)\right]_{p} \cong \operatorname{End}_{R_{p}}\left(E_{p}\right)$ is commutative and $\operatorname{Ann}_{R_{p}}\left(R x_{p}\right)=\operatorname{Ann}_{R_{p}}\left(E_{p}\right)=\left[\operatorname{Ann}_{R}(E)\right]_{p}$. Thus by relationship between primes of $R_{p}$ and primes of $R$ contained in $p$ we get $p \in \operatorname{Min}(\operatorname{Ann}(E))$. So let $p \in \operatorname{Min}(\operatorname{Ann}(x)), x \in E$. Put $T=R_{p}, q=p T, F=E_{p}, y=x / 1 \in F$. $T$ is quasi-local semi-prime with maximal ideal $q=\sqrt{\operatorname{Ann}_{T}(y)}$. By 2.1 there is nonzero $f: F \rightarrow F$ with $f(F) \subset T y$. Let $f(y)=a y$. Then $a \notin q$ else $f$ is nilpotent and consequently zero since $\operatorname{End}_{T}(F)$ is semiprime. Let $b \in q$ and define $h=b a^{-1} f$. Then $h$ is nilpotent since $b \in \sqrt{\operatorname{Ann}(y)}$ and $h(F) \subset T y$. Thus $h=0$. Hence $0=h(y)=b y$. Therefore $q=\operatorname{Ann}_{T}(y)$. Let $M=F / T y$ and suppose $M \neq q M$. Then $M / q M$ is at least one dimensional over $T / q$ so there is a surjection $g: M \rightarrow T / q=T y$. Let $k$ be the composite of the natural map $F \rightarrow M$ followed by $g$. Then $k \in$ End $(F)$ and $k^{2}=0$. Since End $(F)$ is semiprime we get the contradiction $k=0$ and $T y \neq 0$. Therefore $M=q M$; and thus $F=T y$ by Nakayama. Hence $E_{p}=(R x)_{p}$ as required.

THEOREM 2.3. Let $R$ be quasi-regular ring and $E$ a finitely presented $R$-module. If End $(E)$ is semi-prime and if there is an ideal $I \subset R$ such that Ann $(I)=\operatorname{Ann}(E)$, then $E$ is isomorphic to an ideal of $R$.

Proof. By 2.2 Ass $(E)=\operatorname{Min}(\operatorname{Ann}(E))=\operatorname{Min}(\operatorname{Ann}(I))$. Thus each associated prime of $E$ consists of zero divisors of $R$ [2, Theorem 84]. Therefore the natural map $E \rightarrow E \otimes T$ is injective where $T$ denotes the total quotient ring of $R$. Let $F=E \otimes T . \operatorname{End}_{T}(E) \cong$ $\operatorname{End}_{R}(E) \otimes T$ is semi-prime. Since $T$ is absolutely flat, then $F$ is a direct sum $T e_{1} \oplus \cdots \oplus T e_{n}$ of ideals of $T$ each of which is generated by an idempotent $e_{i}$ of $T$ [1, Exercise 18, p. 64]. Let $i \neq j, h \in$ $\operatorname{Hom}_{T}\left(T e_{i}, T e_{j}\right)$. Define $f: F \rightarrow F$ by $f\left(e_{k}\right)=0$ for $k \neq i$ and $f\left(e_{i}\right)=$ $h\left(e_{i}\right)$. Then $f^{2}=0$ and thus $h=0$. Hence the idempotents $e_{i}$ are mutually orthogonal and therefore $F=T e_{1}+\cdots+T e_{n}$ is an ideal of $T$. Now multiplication by a suitable regular element will move the image of $E$ in $F$ inside $R$.

The hypothesis on $\operatorname{Ann}_{R}(E)$ in 2.3 is satified when $E$ is faithful.

There is some evidence that 2.3 may be valid for noncommutative rings. For example if $R$ is an absolutely flat semi-prime ring and $E$ a finitely presented right $R$-module (or more generally a projective right $R$-module) and if End $(E)$ is semi-prime then $E$ is isomorphic to an ideal.

Added March 12, 1973. S. Alamelu has independently obtained Theorem 1.1. Her results will appear in the Proceedings of the American Mathematical Society. 


\section{REFERENCES}

1. N. Bourbaki, Algèbre commutative, Actualitès Sci. Indust. No. 1290, Hermann, Paris, 1961.

2. I. Kaplansky, Commutative rings, Allyn and Bacon, Boston, 1970.

3. W. V. Vasconcelos, On commutative endomorphism rings, Pacific J. Math., 35 (1970), 795-798.

Received December 7, 1971.

University of Puerto Rico, Rio Piedras 



\section{PACIFIC JOURNAL OF MATHEMATICS}

EDITORS

\author{
H. SAMELSON \\ Stanford University \\ Stanford, California 94305 \\ C. R. HOBBY \\ University of Washington \\ Seattle, Washington 98105
}

\author{
J. DUGUNDJI \\ Department of Mathematics \\ University of Southern California \\ Los Angeles, California 90007 \\ RICHARD ARENS \\ University of California \\ Los Angeles, California 90024
}

\section{ASSOCIATE EDITORS}

E. F. BECKENBACH

B. H. NeUManN

F. WOLF

K. YosHIDA

\section{SUPPORTING INSTITUTIONS}

UNIVERSITY OF BRITISH COLUMBIA

CALIFORNIA INSTITUTE OF TECHNOLOGY

UNIVERSITY OF CALIFORNIA

MONTANA STATE UNIVERSITY

UNIVERSITY OF NEVADA

NEW MEXICO STATE UNIVERSITY

OREGON STATE UNIVERSITY

UNIVERSITY OF OREGON

OSAKA UNIVERSITY
UNIVERSITY OF SOUTHERN CALIFORNIA

STANFORD UNIVERSITY

UNIVERSITY OF TOKYO

UNIVERSITY OF UTAH

WASHINGTON STATE UNIVERSITY UNIVERSITY OF WASHINGTON

AMERICAN MATHEMATICAL SOCIETY NAVAL WEAPONS CENTER 


\section{Pacific Journal of Mathematics}

\section{Vol. 45, No. $1 \quad$ September, 1973}

William George Bade, Complementation problems for the Baire classes .......... 1

Ian Douglas Brown, Representation of finitely generated nilpotent groups ........ 13

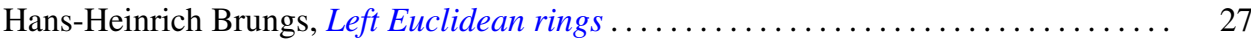

Victor P. Camillo and John Cozzens, A theorem on Noetherian hereditary rings ..... 35

James Cecil Cantrell, Codimension one embeddings of manifolds with locally flat

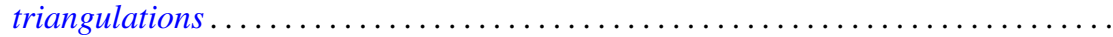

L. Carlitz, Enumeration of up-down permutations by number of rises . . . . . . . . . .

Thomas Ashland Chapman, Surgery and handle straightening in Hilbert cube

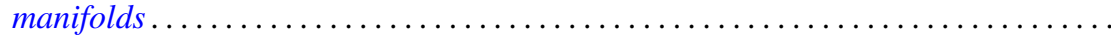

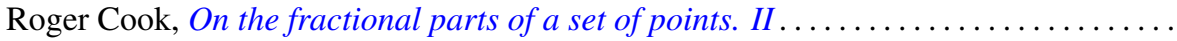

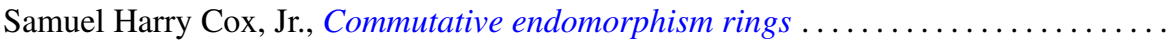

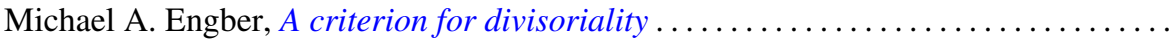

Carl Clifton Faith, When are proper cyclics injective . . . . . . . . . . . . . . 97

David Finkel, Local control and factorization of the focal subgroup . . . . . . . . . 113

Theodore William Gamelin and John Brady Garnett, Bounded approximation by

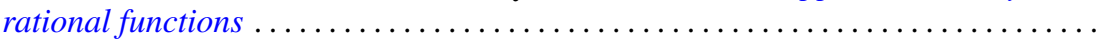

Kazimierz Goebel, On the minimal displacement of points under Lipschitzian

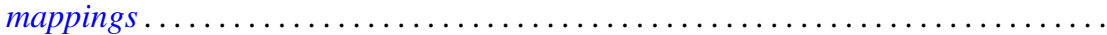

Frederick Paul Greenleaf and Martin Allen Moskowitz, Cyclic vectors for representations associated with positive definite measures: nonseparable

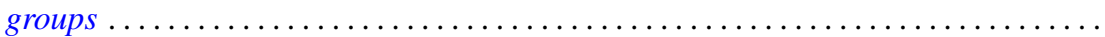

Thomas Guy Hallam and Nelson Onuchic, Asymptotic relations between perturbed linear systems of ordinary differential equations .

David Kent Harrison and Hoyt D. Warner, Infinite primes of fields and completions. .

James Michael Hornell, Divisorial complete intersections . ......

Jan W. Jaworowski, Equivariant extensions of maps ..............

John Jobe, Dendrites, dimension, and the inverse arc function .. .

Gerald William Johnson and David Lee Skoug, Feynman integrals of non-factorable

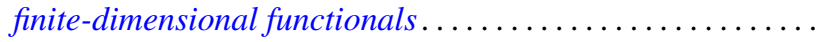

Dong S. Kim, A boundary for the algebras of bounded holomorphic functions ...... 269

Abel Klein, Renormalized products of the generalized free field and its derivatives ... 275

Joseph Michael Lambert, Simultaneous approximation and interpolation in $L_{1}$ and

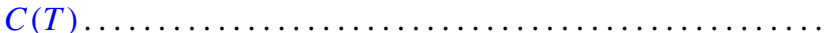

Kelly Denis McKennon, Multipliers of type $(p, p)$ and multipliers of the group

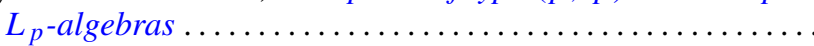

William Charles Nemitz and Thomas Paul Whaley, Varieties of implicative

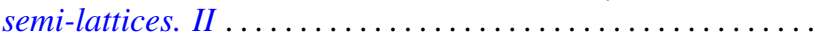

Donald Steven Passman, Some isolated subsets of infinite solvable

Norma Mary Piacun and Li Pi Su, Wallman compactifications on E-completely

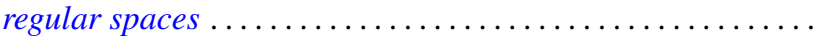

Jack Ray Porter and Charles I. Votaw, $S(\alpha)$ spaces and regular Hausdorff extensions....

Gary Sampson, Two-sided $L_{p}$ estimates of convolution transforms .

Ralph Edwin Showalter, Equations with operators forming a rig
Raymond Earl Smithson, Fixed points in partially ordered sets .

Victor Snaith and John James Ucci, Three remarks on symmetric products and

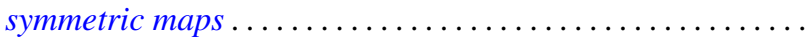

